Final Report for the period

June 30, 2008 March 29, 2009

Submitted by: Stefan Vasile, 617-964-1788, svasile@apeakinc.com

Authors: Dr. Stefan Vasile, aPeak Inc.

Dr. Zheng Li, Brookhaven National Laboratory

Prime contractor: APeak Inc., 77 Oak St, Suite 203, Newton, MA 02464

Subcontractor: Brookhaven National Laboratory

PREPARED FOR THE UNITED STATES DEPARTMENT OF ENERGY, OFFICE OF HIGH ENERGY PHYSICS

\title{
Distribution Statement
}

\section{LEGAL DISCLAIMER}

This report was prepared as an account of work sponsored by an agency of the United States Government. Neither the United States Government nor any agency thereof, nor any of their employees, nor any of their contractors, subcontractors or their employees, makes any warranty, express or implied, or assumes any legal liability or responsibility for the accuracy, completeness, or any third party's use or the results of such use of any information, apparatus, product, or process disclosed, or represents that its use would not infringe privately owned rights. Reference herein to any specific commercial product, process, or service by trade name, trademark, manufacturer, or otherwise, does not necessarily constitute or imply its endorsement, recommendation, or favoring by the United States Government or any agency thereof or its contractors or subcontractors. The views and opinions of authors expressed herein do not necessarily state or reflect those of the United States Government or any agency thereof. 


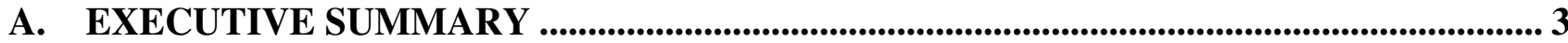

A.1 CONTRIBUTION TO THE DEVELOPMENT OF HIGH TRACKING RESOLUTION DETECTORS............ 3

A.2 TECHNICAL EFFECTIVENESS AND FEASIBILITY …........................................................ 3

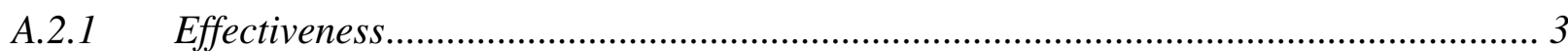

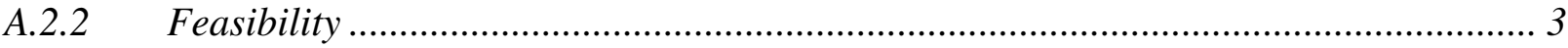

A.3 QUESTIONS PLANNED TO ANSWER DURING PHASE I RESEARCH..................................... 3

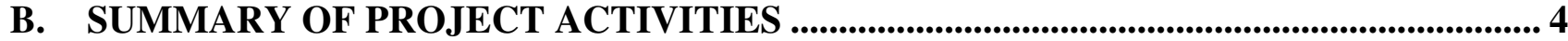

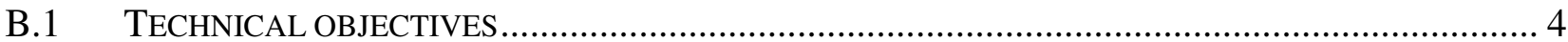

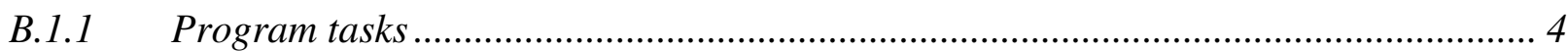

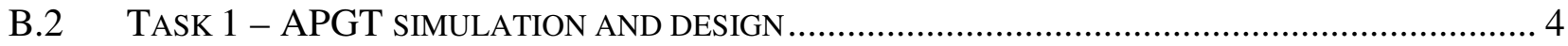

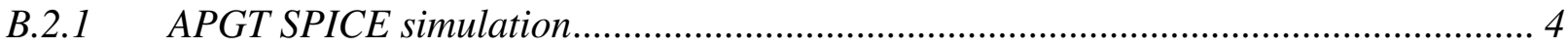

B.2.2 APGT SPICE simulation verification through hybrid APGT prototyping.................. 6

B.2.2.1 Prototype APGPT pixel assembly and measurements......................................... 6

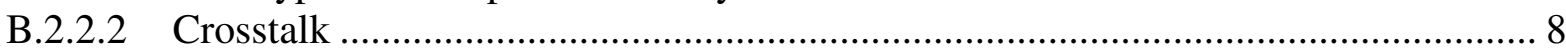

B.3 TASK 3: EVALUATION OF THE RADIATION DAMAGE AND ANNEALING ................................ 9

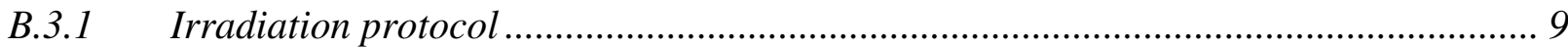

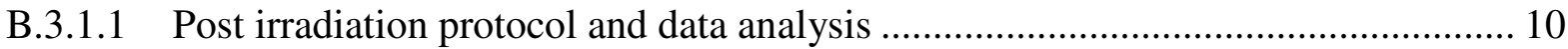

B.3.1.2 Damage annealing.................................................................................. 13

B.3.1.3 Detection efficiency ................................................................................ 13

B.3.2 DLTS Results at Brookhaven National Laboratory $(B N L)$................................... 15

B.4 MAIN CONCLUSIONS OF THE PHASE I .......................................................................... 18

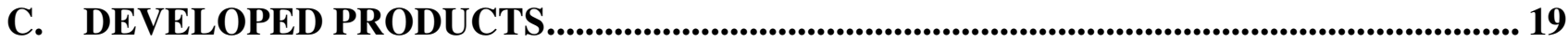

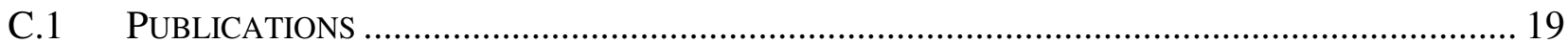

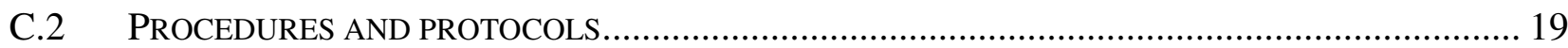

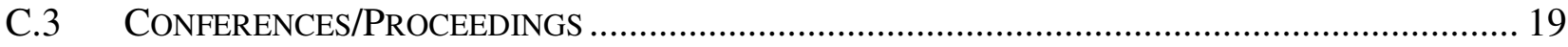




\section{A. EXECUTIVE SUMMARY}

\section{A.1 Contribution to the development of high tracking resolution detectors}

In our Phase I proposal we discussed the advantages and disadvantages of using Geiger Photodiode (GPD) junctions for micro tracking. The most leveraging advantage of using the Active Pixel Geiger Tracker (APGT) concept is that the data output is in binary format, which allows much faster response than the conversion time achieved in multiplexed ADCs or pixel level ADCs $(25 \mu \mathrm{s})^{1}$. Indeed, the GPD detects and converts the charge to a binary signal in nanoseconds and it needs additional 50-100 ns to reset by itself or few nanoseconds if controlled by active reset.

\section{A.2Technical effectiveness and feasibility}

\section{A.2.1 Effectiveness}

The overall goal of the program was to develop APGT arrays for microvertex applications with improved readout rate, simplified signal processing and capable of readout, charge conversion and reset functions at pixel level. The goal of Phase I program was to develop silicon APGT tracking detectors with high gain, calorimetric output and fast readout rate capable of short radiation length with application in high resolution tracking needed in Nuclear Physics and High Energy Physics research. The technical approach is to fabricate backside-illuminated Geiger segmented pixels with limited calorimetric output and monolithically integrated readout at pixel level using standard CMOS and thin the backside to less than 50 microns in such a way to minimize the probability of pair collection and/or generation by long range, hot electrons. The program benefited from previous expertise at aPeak Inc. on microvertex detectors with binary output, radiation evaluation of such detectors, as well as expertise with radiation damage evaluation at the Brookhaven National Laboratory (BNL).

The program contributed with a new readout circuit and pixel architecture to the engineering of high-resolution particle tracking arrays and has demonstrated high detection efficiency even after $20-30 \%$ residual degradation of the charge collection efficiency.

\section{A.2.2 Feasibility}

\section{A.3 Questions planned to answer during Phase I research}

Over the last two years, our R\&D effort has resulted in a fast progress on improving our Geiger array technology performance, verifying their reliability and developing a large cell library. Much progress has been supported by recent funding from the U S DOE Nuclear Physics division to evaluate the feasibility of using the Geiger avalanche for microvertex detectors. The expertise gained and technologies developed or qualified during that program provided the technological basis and expertise to propose and carry out the work planned in this program. The development of APGT arrays for high-resolution tracking raises new questions related to the minimum pixel pitch and integration issues. In Phase I we planed to answer the following questions related to the APGT pixel structure, readout design and performance, and better understand the radiation damage and annealing in these detectors:

1. What is the minimum pixel pitch at the optimal bias?

2. What is the optimal sub-pixel layout and number of sub-pixels/APGT pixel

3. What is the optical and electrical crosstalk at the optimal bias? 
4. How to integrate the readout at pixel level?

5. Is there amplification needed at pixel level?

6. How to assess the radiation damage and annealing in APGT test pixels?

7. What is the track resolution if using track centroiding?

\section{B. SUMMARY OF PROJECT ACTIVITIES}

\section{B.1 Technical objectives}

This Phase I program is based on the technology previously developed at aPeak for thin, backside illuminated tracking detectors mounted on flexible substrates to yield low radiation length high assembly. Therefore Phase I technical objectives will be focused on developing the segmented pixel structure and the readout in calorimetric mode.

In Phase I we will evaluate and demonstrate the main elements of the APGT array technology and measure its performance, namely sensor structure, readout/ reset cell architecture, as well as radiation/damage and annealing. The Phase I technical objectives are:

(1) Design pixel layout and readout cells;

(2) Fabricate small APGT arrays (containing a variety of test blocks) to help:

(a) select the readout cell layout;

(b) evaluate and identify methods to decrease the crosstalk;

(d) identify operation conditions to help design sub-pixel resolution enhancement algorithms;

(4) Evaluate the radiation damage and damage annealing.

\section{B.1.1 Program tasks}

Phase I program comprised of the following tasks:

Task 1: APGT simulation and design

Task 2: APGT fabrication and performance evaluation; and

Task 3: Evaluation of the radiation damage and annealing.

\section{B.2 Task 1 - APGT simulation and design}

Technical challenges toward integration of high-resolution APGT pixel arrays are: (1) optical crosstalk; and (2) the guard ring to protect the pixel against premature breakdown.

\section{B.2.1 APGT SPICE simulation}

Single Geiger junctions are single-carrier binary devices. Electrons or holes crossing the Geiger avalanche junction may trigger a Geiger avalanche pulse at $10^{8}$-charge gain. As the Geiger avalanche develops within few nanoseconds, the Geiger current may exceed $1 \mathrm{~mA} .\left(10^{8} \overline{\mathrm{e}} \mathrm{x} 1.6 \mathrm{e} 10^{-}\right.$

$\left.{ }^{19} \mathrm{C} / 10^{-8} \overline{\mathrm{e}} \mathrm{sec}\right)$. The Geiger detector during avalanche is modeled like a switch in series with a current path, bypassing the reverse biased junction. In a practical configuration, a resistor in series with the Geiger junction protects the junction against destructive breakdown. The current flowing through the series resistor drops the voltage on the Geiger junction below the breakdown voltage and the Geiger avalanche is quenched (the resistor is known as the passive quenching resistor). As shown in Figure 1 we model the Geiger detector as a p-n junction reverse biased diode in parallel with a Geiger current bypass controlled by a parallel resistor in series with a voltage controlled switch S1. Switch S1 opens when the voltage on the p-n junction drops below the breakdown voltage thus quenching the Geiger avalanche. Switch X1 is a time controlled switch simulating the onset of the Geiger avalanche. 


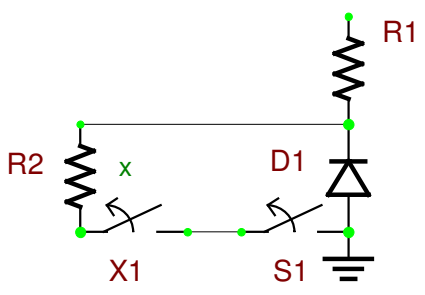

Figure 1. Equivalent circuit for single Geiger diodes.

Single APGT pixels contain an array of such junctions each protected with its own passive quenching resistor. In Phase I we simulated and fabricated APGT pixels each containing a matrix of $3 \times 3$ Geiger junctions with integrated passive resistors. Though this is not a conceptual limitation, we believe that the dynamic range offered by 9-junction APGT pixels is suitable for the extraction of the position of the track. Phase II will analyze the tradeoffs between dynamic range, APGT pixel size and resolution.

The Geiger signal generation was simulated in Spice using 9 multiplexed Geiger junction pixels. The equivalent circuit of the APGT pixel and readout cells is shown in Figure 2. The SPICE output of a 9-junction APGT pixel is shown in Figure 3.

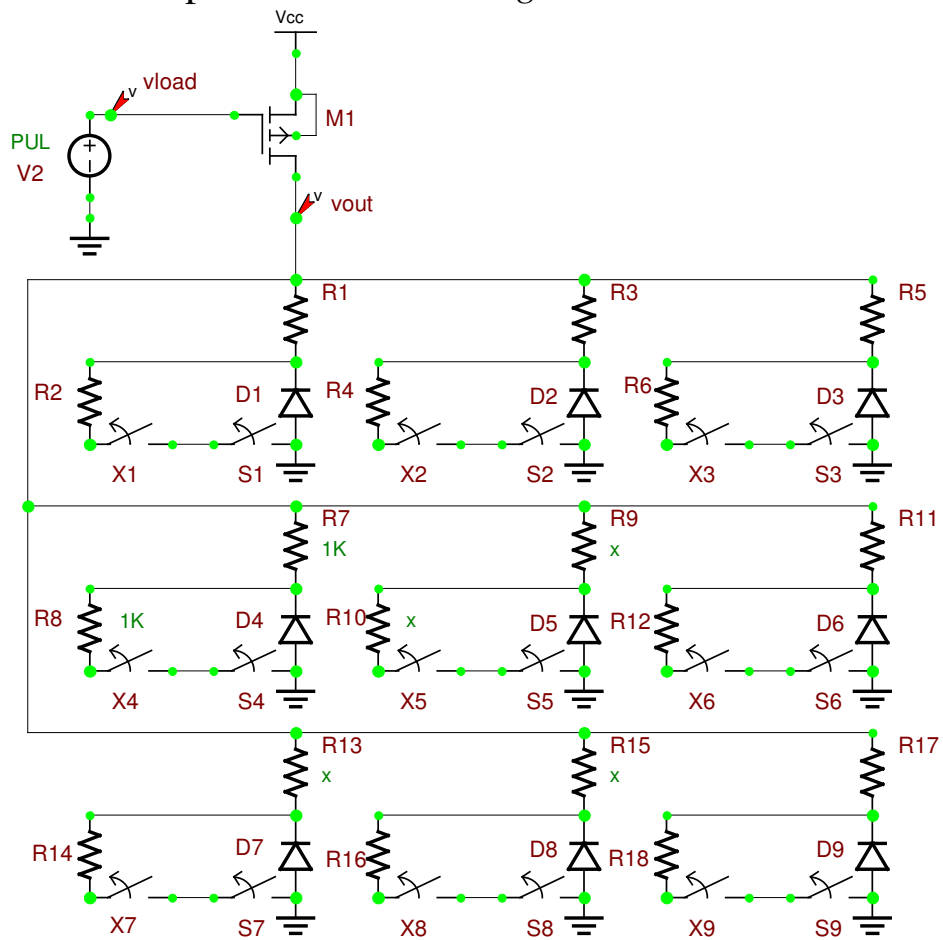

Figure 2. Equivalent circuit of the 9-junction APGT pixel.

Three events with different number of Geiger events were simulated. At $t=0$, the APGT pixel is biased above the breakdown voltage at Vcc and the transistor M1 (PMOS) is turned OFF. A nuclear event is simulated by firing a variable number of Geiger junctions (here 6, 2 and 1 junctions). The number of fired junctions is proportional with the number of electrons generated by the charged particle and collected by the APGT pixel. The slope of the trailing edge signal output $\mathrm{dVout} / \mathrm{dt}$ is proportional with the number of fired junctions and hence with the number of electrons generated by the charged particle. The current flowing through the passive quenching resistors 
drops the voltage on the Geiger junctions below the breakdown voltage and opens some of the X1X9 switches, thus quenching the Geiger avalanche. Once the Geiger current is quenched, the APGT pixel remains charged at a voltage slightly below the breakdown voltage. The APGT is charged back to Vcc by an active Vload on M1 gate. The APGT slope of the Vout signal increases with the number of electrons/pulse.

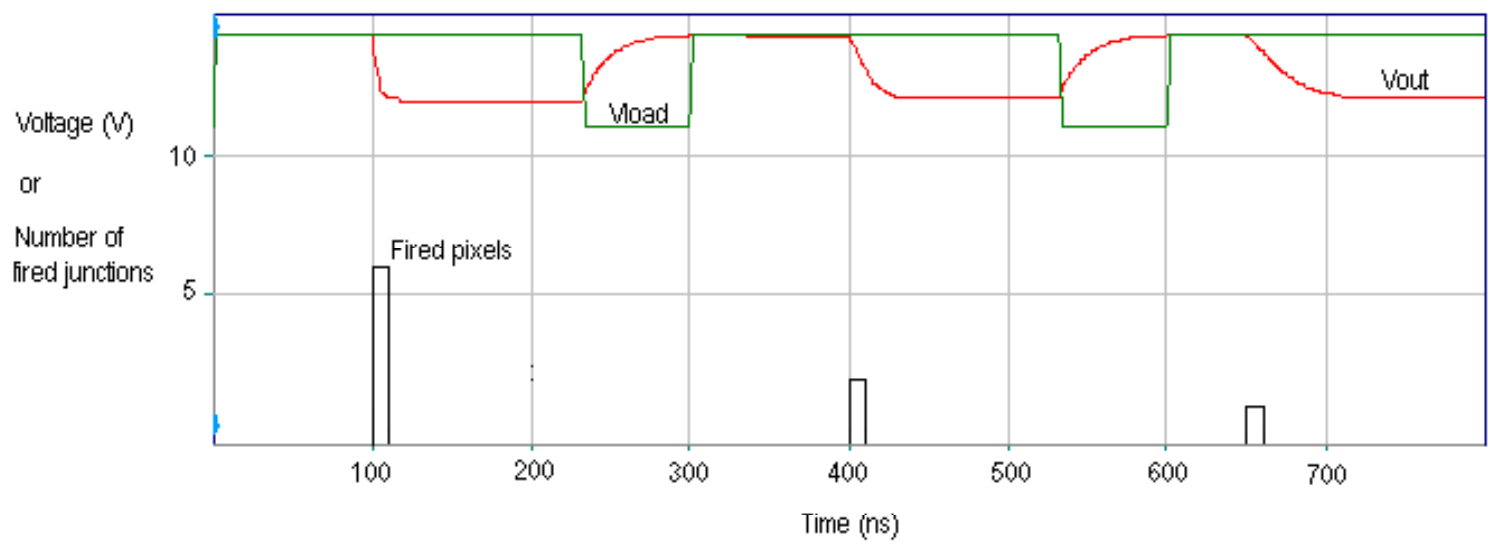

Figure 3. SPICE simulation of the proposed differential operation.

\section{B.2.2 APGT SPICE simulation verification through hybrid APGT prototyping}

\section{B.2.2.1 Prototype APGPT pixel assembly and measurements}

APGT pixels were assembled out of nine discrete Geiger junctions. Vload was generated by a tristate output and Vout was fed to a unity gain amplifier to account for the capacitive load of the oscilloscope used for displaying the waveforms. Charged particle detection events were simulated using a pulsed 1060 nm, 30ps laser with constant intensity (shown in Figure 4 bottom left). The number of firing junctions in the APGT was varied by applying an increasing bias on the APGT pixel. Figure 5 shows the waveforms at the input of the amplifier with the laser beam OFF (a,c) and ON (b,d) for two applied voltages on the APGT pixel. The waveform shape shows the modulation of the trailing edge with the number of junctions undergoing a Geiger avalanche detection event and is similar with the Spice simulation results presented in section Figure 3- the difference is that the steady state plateau in the acquired waveforms is reached slower due to the finite capacitance at the input of the unity gain amplifier. The plateau voltage corresponds to the breakdown voltage (below the breakdown voltage the resistance of the Geiger junction increases to about $13 \mathrm{~V} / \mathrm{pA}(13 \mathrm{G} \Omega)$ and the Geiger junction capacitance remains charged slightly below the breakdown voltage. Data shown in Figure 5b indicate that APGT are capable of sub-microsecond time response. 
Peak

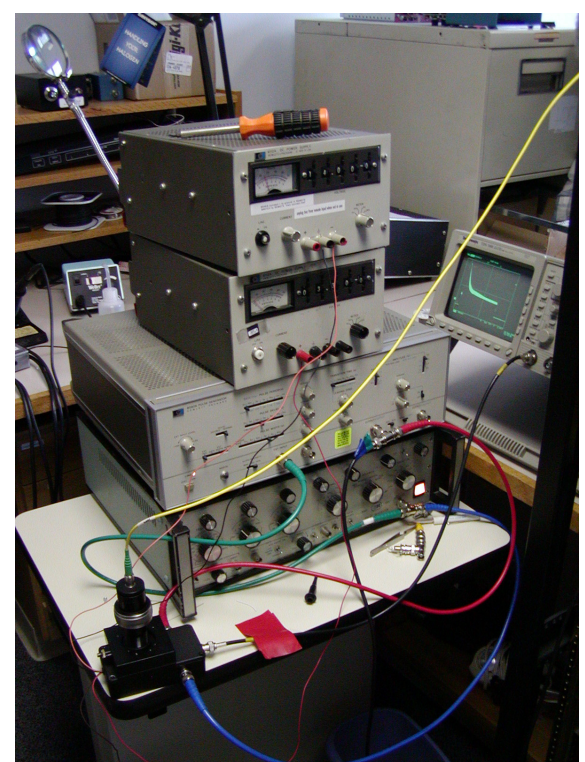

Figure 4. Setup used to simulate MIP charge events ( the device under test is shown at the bottom left with the yellow fiber optic emitting 30 ps $1060 \mathrm{~nm}$ photons.
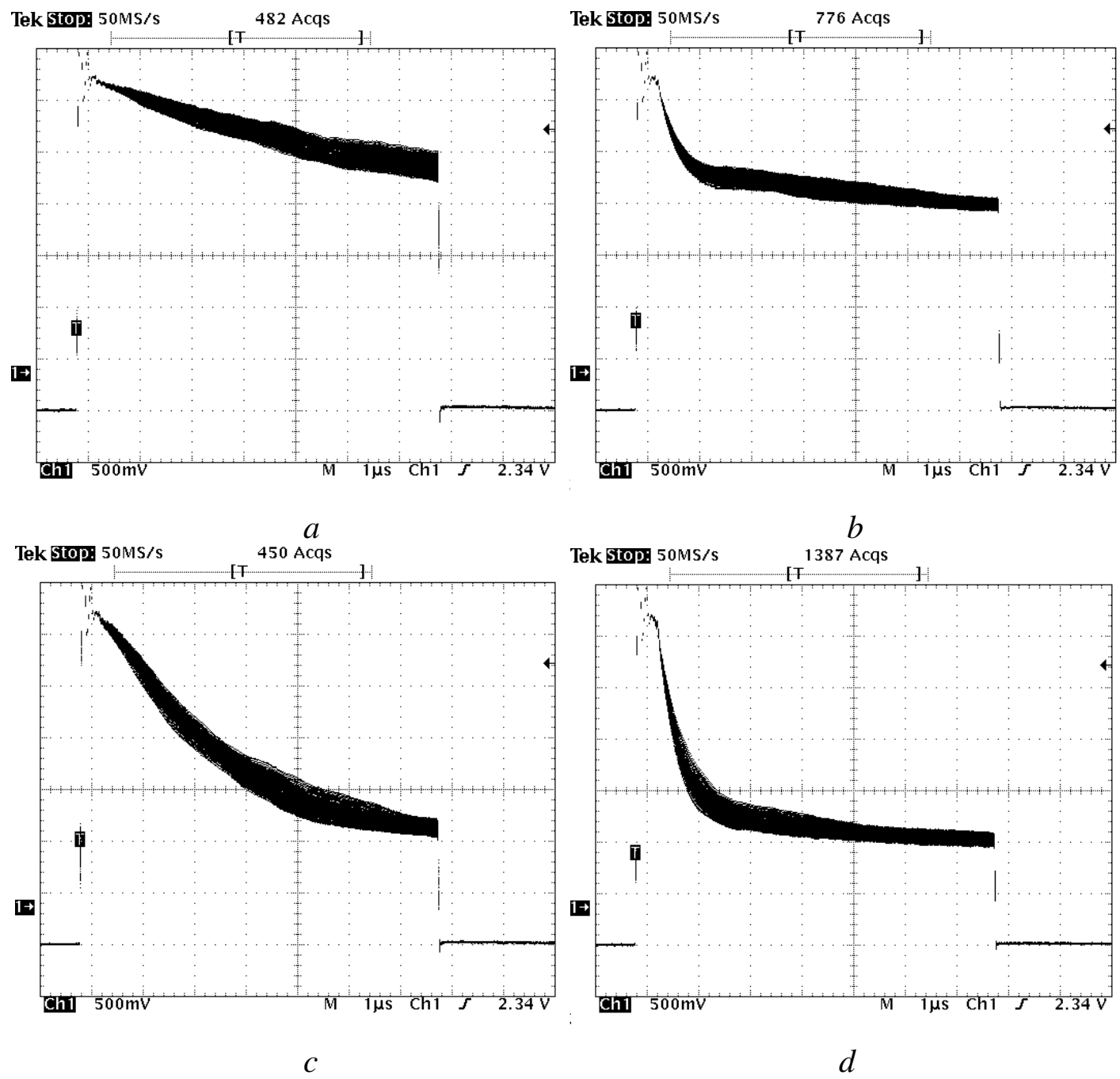
Figure 5. Multiple waveforms at the APGT output, acquired for two bias conditions on APGT with pulsed laser OFF/ON. (a) $V_{A P G T}=13.4 \mathrm{~V}$, Laser OFF, (b) $V_{A P G T}=13.4 \mathrm{~V}$, Laser ON, (c) $V_{A P G T}=13.6 \mathrm{~V}$, Laser $O F F$, (d) $V_{A P G T}=13.6 \mathrm{~V}$, Laser $O N$.

The data in Figure 5 show that the slew rate of the trailing edge is dependent on the charge injected into the 9-junction APGT pixel and indicates that the signal has to be differentiated in order to yield a signal proportional to the charge deposited into the 9-junction pixel. Next, the output of the APGPT was routed to a differentiating amplifier as shown in Figure 6. The APGPT output for an increasing number of fired junctions is shown in Figure 7.

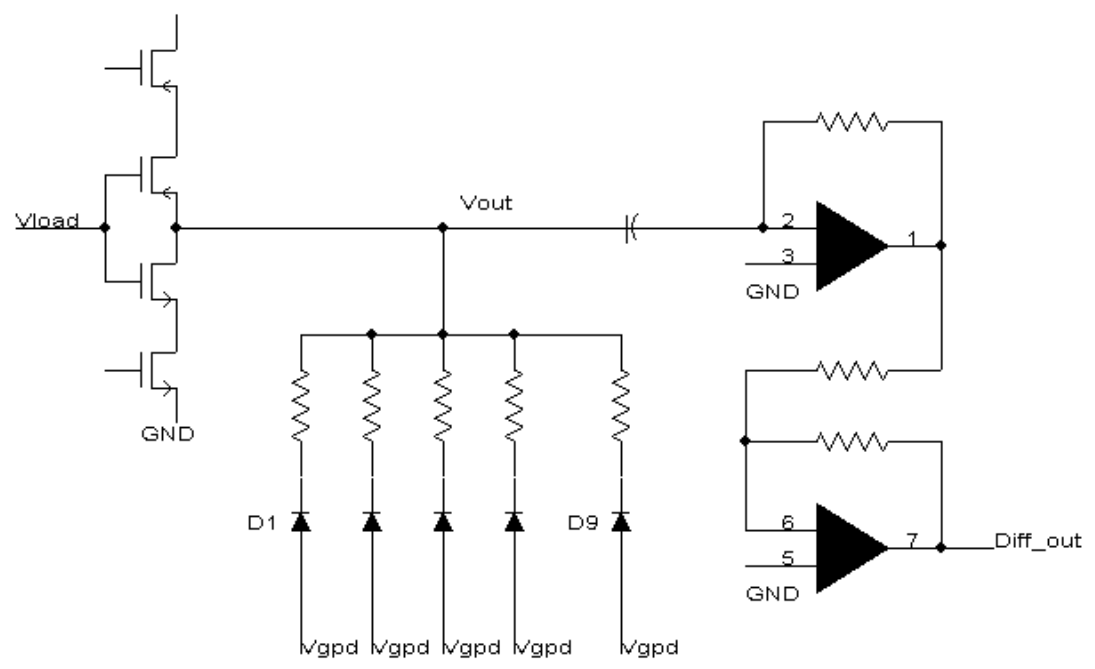

Figure 6. APGPT in differential mode.

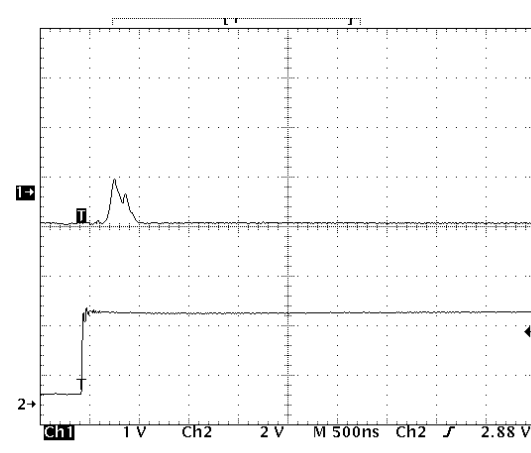

$a$

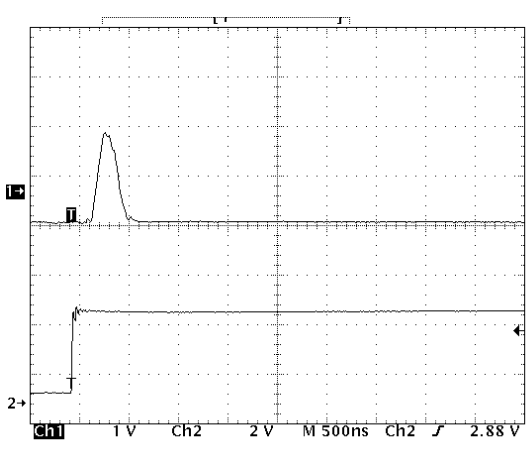

$b$

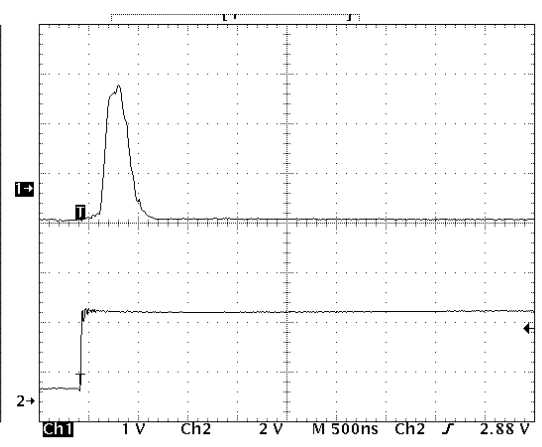

$c$

Figure 7. APGPT outputs acquired in differential mode for a variable (increasing) number of charges induced optically into the pixel. The differential output of the APGT breadboarded pixel shows the signal output increasing with the number of fired Geiger junctions. Channel 2 provides the gate reference from the laser driver output.

\section{B.2.2.2 Crosstalk}

Geiger detector arrays may exhibit large crosstalk between adjacent pixels due to secondary photons emitted after the thermalization of hot carriers generated during the avalanche process. The crosstalk increases with the bias applied above the breakdown voltage. Fortunately, MIPs 
generate in thin APGTs an excessive charge, thus allowing APGPT operation at lower bias with no penalty to the detection efficiency. In order to identify whether APGTs biased at lower voltage may exhibit low crosstalk we investigated the dependence of the crosstalk on distance and applied bias. Prior detection efficiency measurements have shown that an overbias of only $100 \mathrm{mV}$ will allow $100 \%$ detection efficiency. Figure 8 shows that for $100 \mathrm{mV}$ overbias the crosstalk at $20 \mathrm{um}$ pitch is close to $1 \%$.

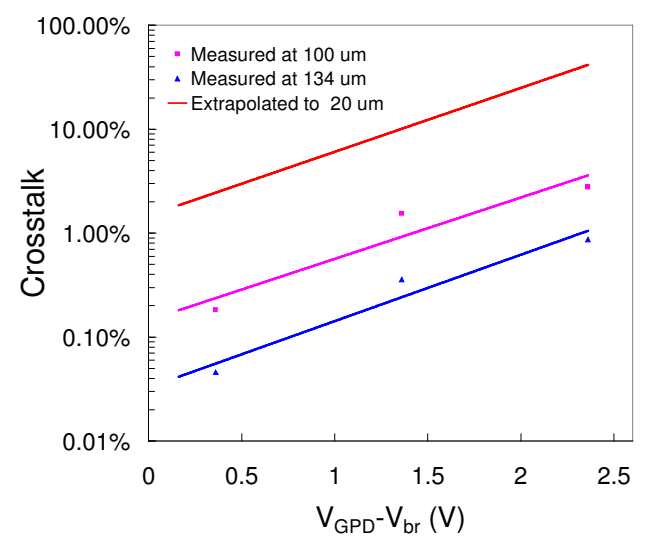

Figure 8. Dependence of crosstalk on the applied bias above the breakdown voltage (experimental) and extrapolated crosstalk for 20-micron pitch (upper, red line) indicate that at 0.2 $V$ bias the crosstalk is only $2 \%$.

\section{B.3 Task 3: Evaluation of the radiation damage and annealing}

\section{B.3.1 Irradiation protocol}

During a previous program we irradiated the APGT arrays with protons at LBNL, Cyclotron 88". Due to the long deactivation time, the samples irradiated at the highest dose required a time longer than Phase I duration. After consulting with Dr. Howard Wieman (BNL) we learned that neutron irradiation results in much shorter de activation time and the damage results are equally relevant for this program. Consequently, we used neutron irradiation at the Van de Graff accelerator at $\mathrm{U}$ Lowell. Irradiation dose ranged from $8.8 \times 10^{11}$ to $10^{13}$ neutrons $/ \mathrm{cm}^{2}$. Samples were irradiated in a single run (see Figure 9) - the dose was calculated based on the neutron distribution previously calibrated by the accelerator personnel (neutron distribution is shown in Figure 10). 


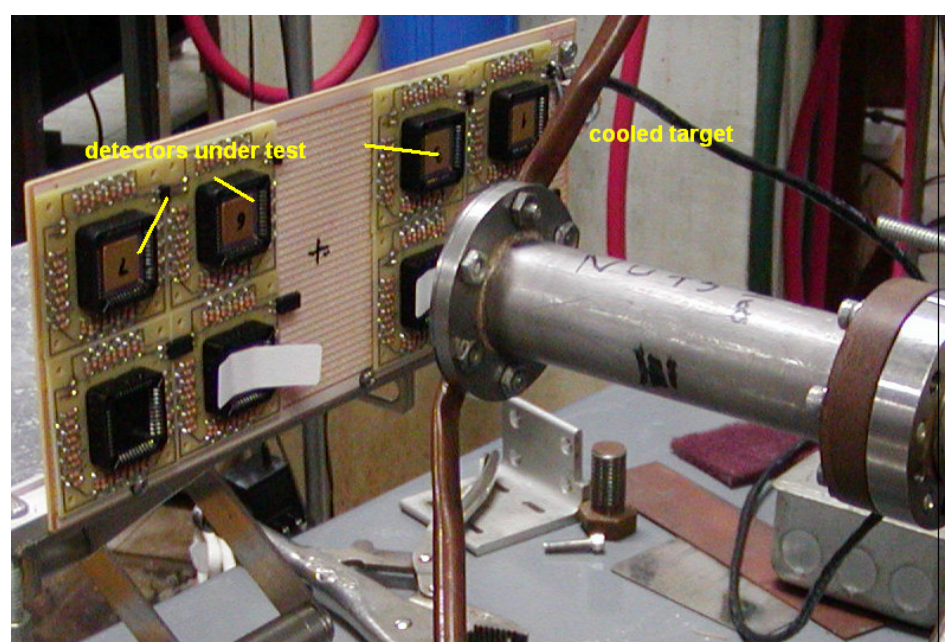

Figure 9. Irradiation setup with devices under test.

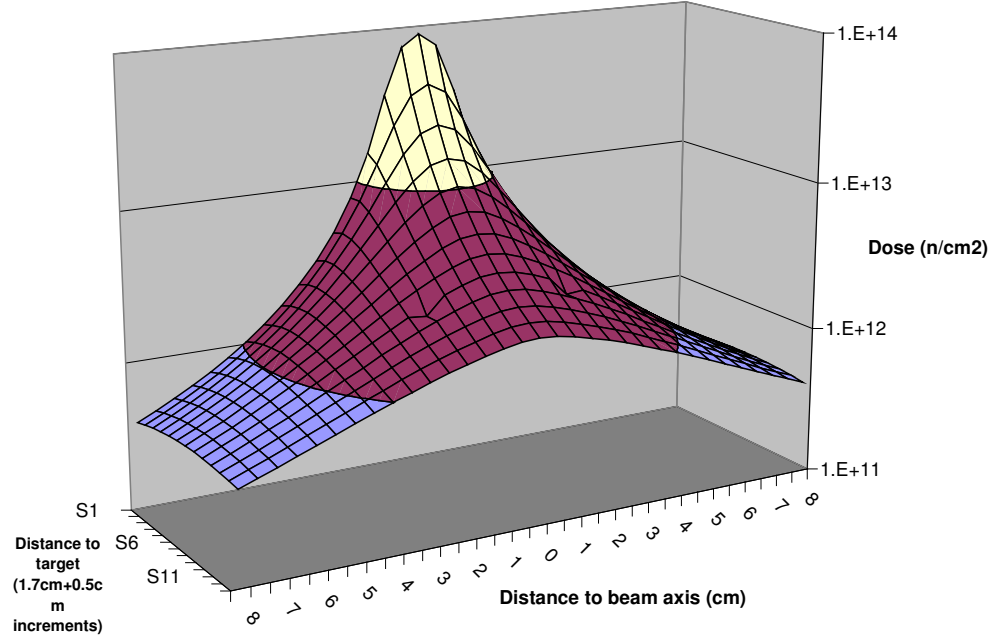

Figure 10. Neutron distribution at the Van de Graff accelerator.

\section{B.3.1.1 Post irradiation protocol and data analysis}

Pre and post irradiation dark count rate (DCR) was used as an indicator of damage creation, while pre irradiation and post annealing DCR was used as an indicator of damage annealing following the heat treatment. Figure 11 shows DCR before and after irradiation.

Figure 12 and b show the DCR degradation probability: at $90 \%$, the maximum DCR damage is less than 100X for the highest dose. The breakdown voltage has degraded insignificantly even after exposure to the highest neutron dose of $10^{13} \mathrm{n} / \mathrm{cm}^{2}$ (Figure 13). 
Prelrradiation Plot

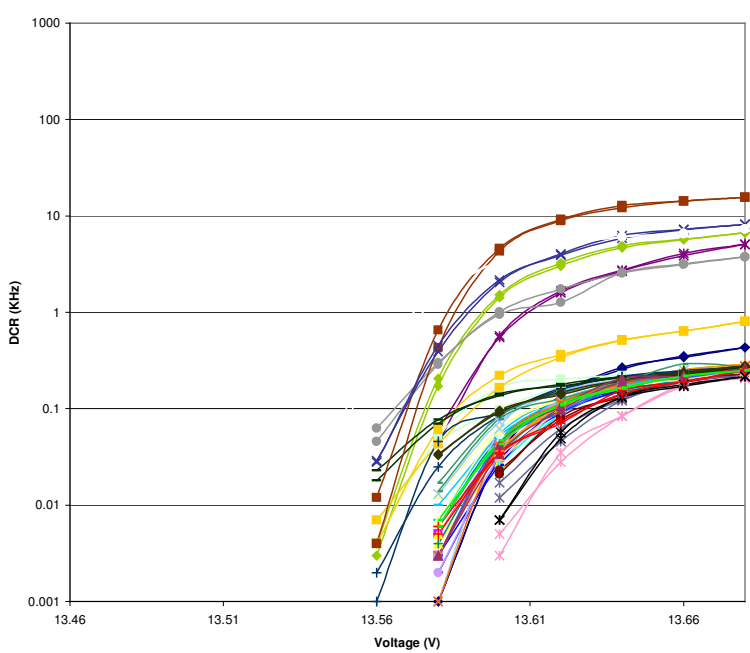

Postlrradiation Plot

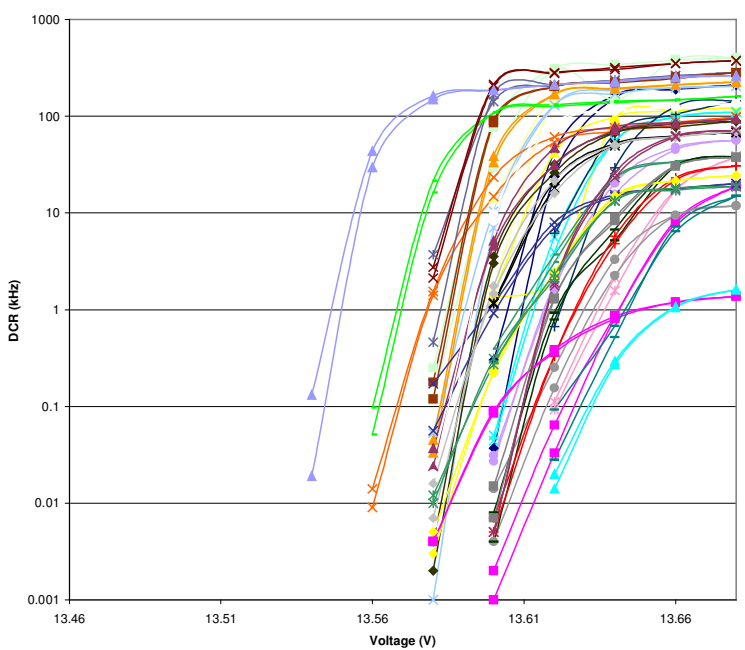

Figure 11. Plots of the measured dark count rates on 36 devices under test (DUT) before (a) and after irradiation with protons at $1 \times 10^{13} \mathrm{n} / \mathrm{cm}^{2}$. All Geiger junctions were operational even after exposure to the highest dose of neutrons (no failed devices).

\section{1e13 n/cm2 DCRpost-DCRpre}

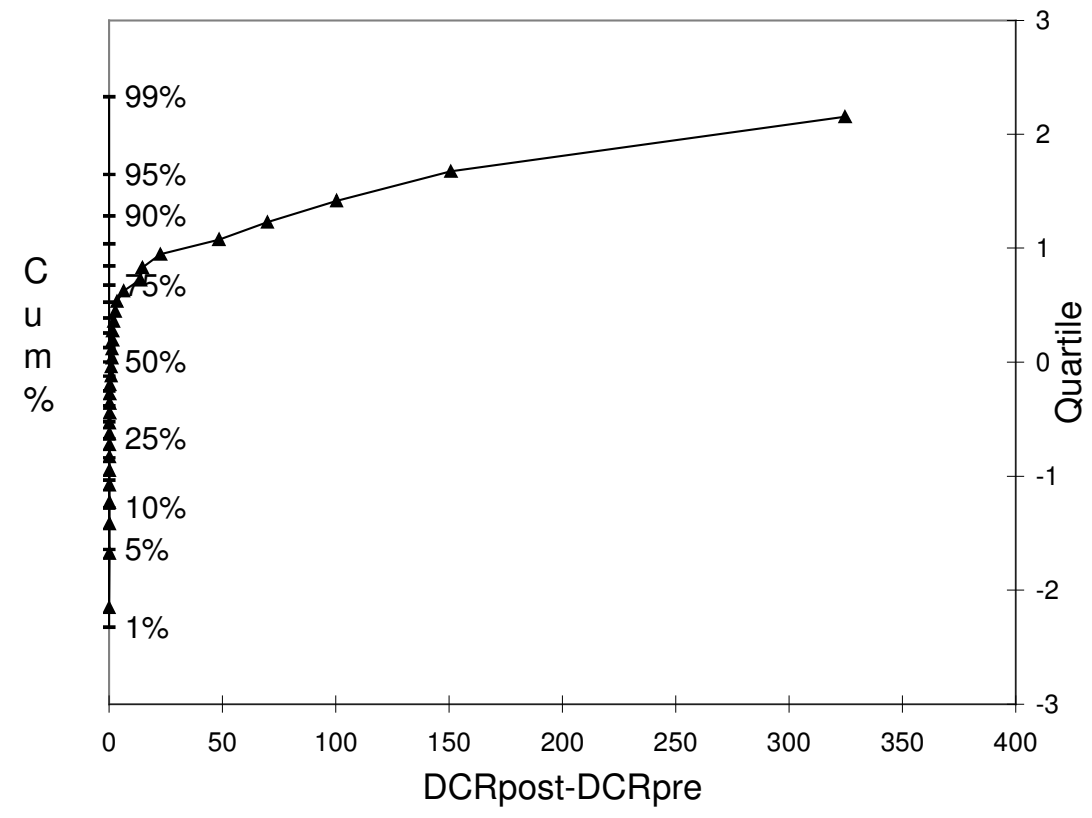

$a$ 


\section{$1 \mathrm{e} 13$ n/cm2 DCRpost-DCRpre}

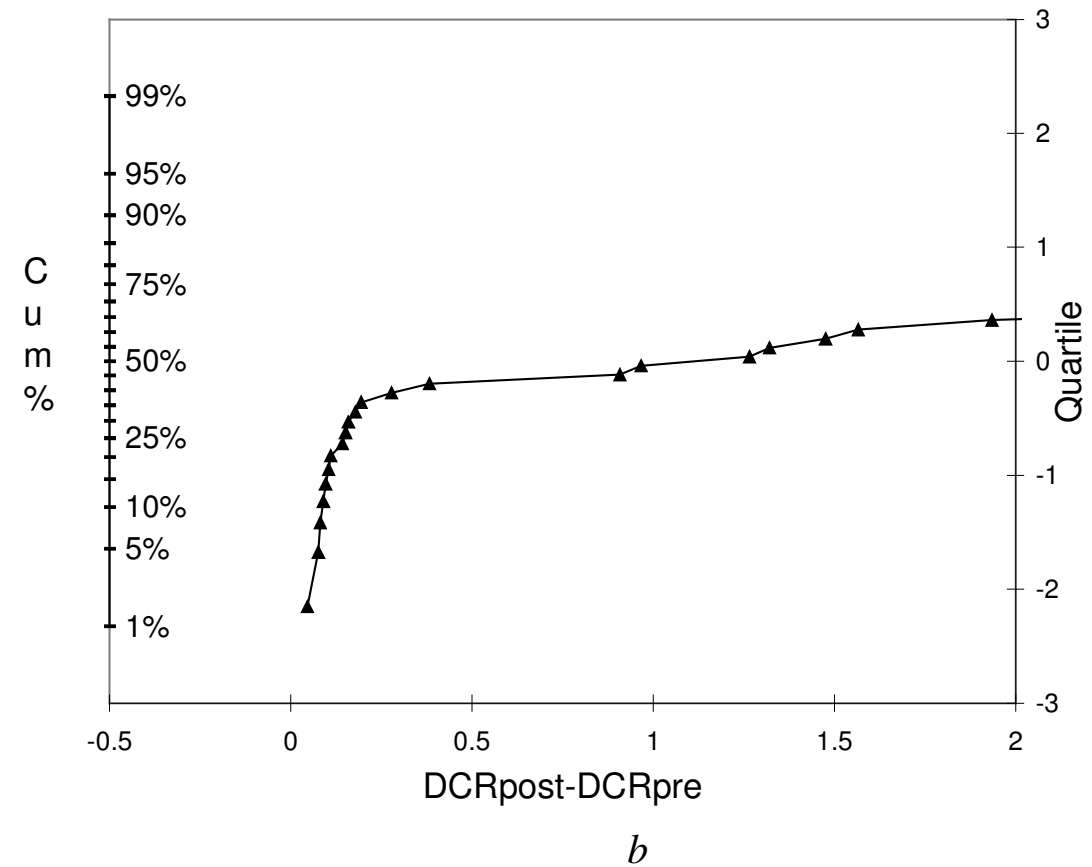

Figure 12 DCR relative increase rate after irradiation with neutrons at $1 \times 10^{13} \mathrm{n} / \mathrm{cm}^{2}:$ (a) full scale: (b) zoom in for comparison with DCR recovery after annealing.

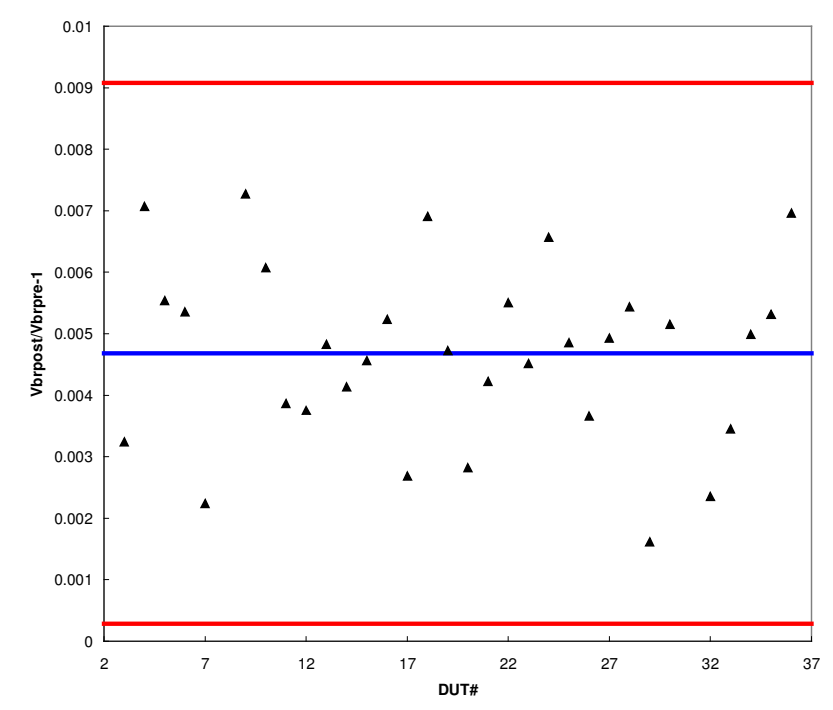

Figure 13. Breakdown voltage relative shift after irradiation at the highest dose $\left(1 \times 10^{13} \mathrm{n} / \mathrm{cm}^{2}\right)$ shows a variation less than $5 \mathrm{mV}$. 
By comparison with previous irradiation experiments with protons on the same type of APGT structures (showing a decrease of the breakdown voltage by $200 \mathrm{mV}$ at only $3 \times 10^{11} \mathrm{p} / \mathrm{cm}^{2}$ (Table 14), irradiation with neutrons does not change significantly the breakdown voltage..

\begin{tabular}{|l|l|l|l|}
\hline T=23C & Sample size & Mean & Std deviation \\
\hline No irradiation & 25 & 13.956 & 0.020 \\
\hline $1 \times 10^{11} \mathrm{p} / \mathrm{cm}^{2}$ & 25 & 13.808 & 0.023 \\
\hline $3 \times 10^{11} \mathrm{p} / \mathrm{cm}^{2}$ & 25 & 13.761 & 0.019 \\
\hline
\end{tabular}

Table 14. Breakdown voltage measured in previous irradiation experiments with protons.

\section{B.3.1.2 Damage annealing}

The damage-annealing schedule was performed at 150C for 24 hours. Date indicated the damage recovery (return to approximately the $=$ same dark count rates measured before irradiation. A probability plot of the difference between DCR after annealing and DCR before irradiation is shown in Figure 15. Note the damage annealing as compared to data in Figure 12. The number of DUT is still 36 GPD junctions.

\section{$1 \mathrm{e} 13$ n/cm2 DCRanneal-DCRpre}

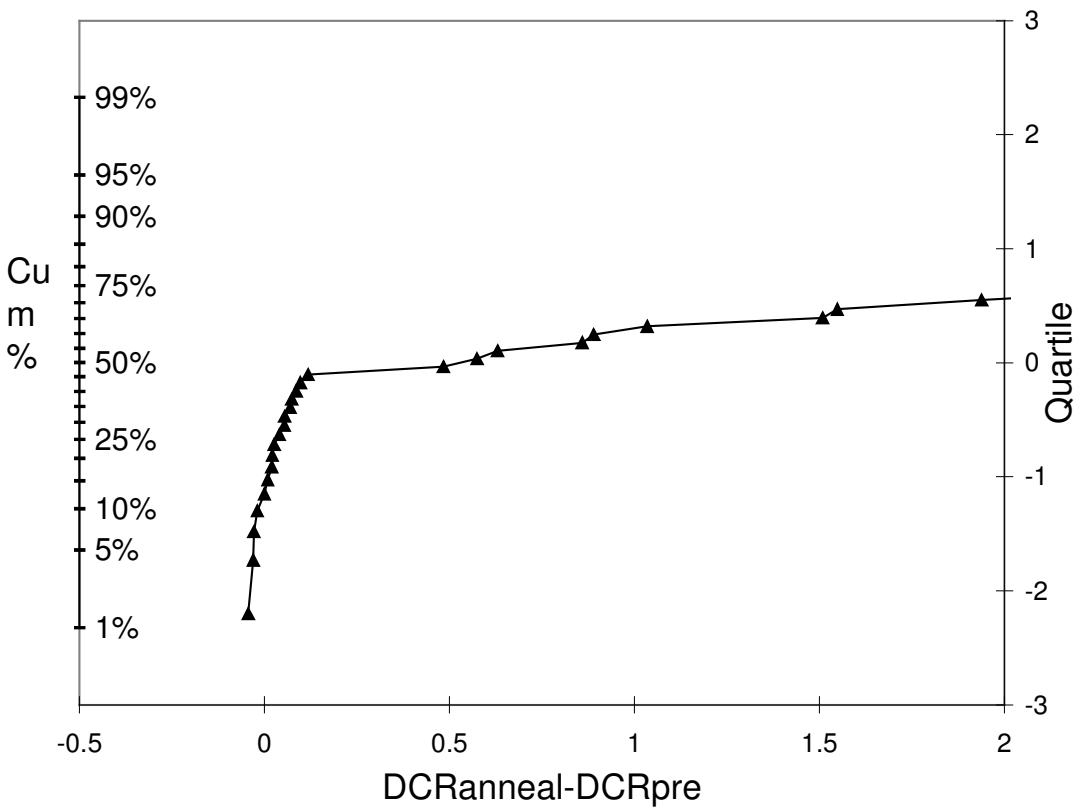

Figure 15. DCR relative change rate after annealing of irradiated samples with neutrons at $1 x$ $10^{13} \mathrm{n} / \mathrm{cm}^{2}$.

\section{B.3.1.3 Detection efficiency}

In a previous work, we have demonstrated that pulsed $1060 \mathrm{~nm}$ photons accurately simulate the charge depth profile by MIPs ${ }^{2}$ (better than 0.999 linear correlation). The measurement setup is using a $1060 \mathrm{~nm} \mathrm{CW}$ laser diode, spatially filtered and focused through the camera port of a epi microscope on test GPD pixels with 13-micron diameter (shown in Figure 16). MIPs generate in silicon 80 e-h pairs /micron. For 50-micron silicon layers, this results in 4,000 e-h pairs. As APGT 
pixel calculated quantum efficiency at $1060 \mathrm{~nm}$ is $2 \%, 80$ e-h pairs will reach the GPD junction and may trigger the Geiger avalanche. For our APGT structure, MIPs are mainly absorbed is the ptype substrate $\mathrm{Si}$. Therefore, the detection efficiency will be controlled by the number of electrons reaching the Geiger avalanche junction. If $P_{e}$ is the single-electron detection efficiency, the detection efficiency $D E$ for $N_{e}$ electrons reaching the junction is:

$D E=1-\exp \left(-P_{e} \times N_{e}\right)$

Single carrier detection efficiency in Geiger junctions is $0.17-0.20$. Therefore the detection efficiency for 80 electrons is:

$D E_{80}=1-\exp (-80 * 0.2)=100 \%$

Single Geiger junction detection efficiency is therefore practically $100 \%$. The degradation after irradiation with protons has shown a $50 \%$ loss in detection efficiency that could be partially recovered to $70-80 \%$ by thermal annealing. Because our APGT design is composed of (at least) nine pixels, the composite detection efficiency is $=1-\exp (-0.7 * 9)=99.8 \%$.

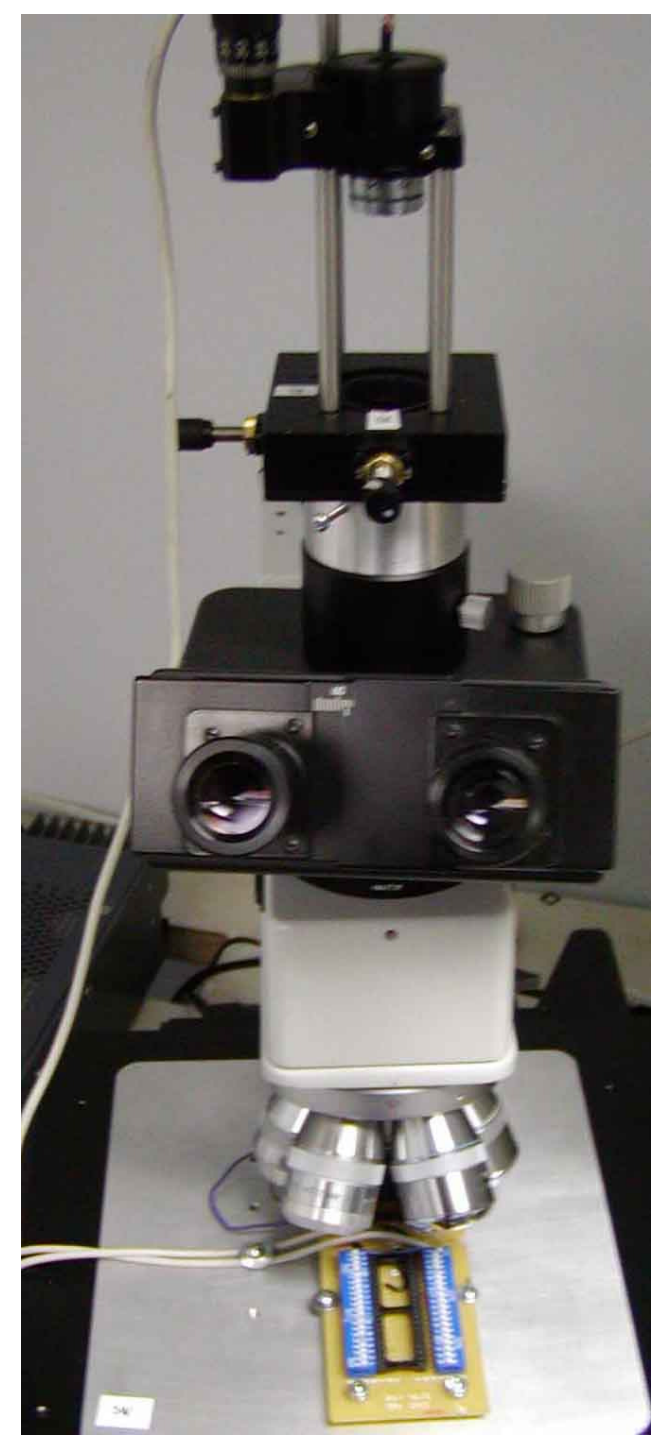

Figure 16. Setup used to measure the detection efficiency. The laser beam is spatially filtered, fed through the photo port of the microscope and then focused on the GPD located on the microscope X-Y stage ( reference photodiode, mounted to the microscope stage is used to monitor the laser power stability - photodiode is located behind the objective turret and is not visible in this picture). 


\section{B.3.2 DLTS Results at Brookhaven National Laboratory (BNL)}

To study the radiation-induced deep levels in the Si device, Dr. Zheng Li at BNL has employed IDLTS system (Current-Deep-Level-transient-Spectroscopy). As shown in Figure 17, the main components of BNL's I-DLTS system are: a cryogenic cooling system consisting of a He cryostat $(10-350 \mathrm{~K})$ with temperature controller SI9650; an illumination system for defect filling consisting of a pulse generator HP 8110A that drives lasers with various wavelengths $(660 \mathrm{~nm}$ to $1030 \mathrm{~nm}$ ); a Keithley 487 power supply to provide bias voltages to the samples; a Keithley 428 current amplifier to amplify the current transient signal obtained from the charges emitted from filled defect levels; and a Tektronix 7704A oscilloscope to record the current transients. In this research, the electron filling of the defect levels is achieved by an $822 \mathrm{~nm}$ IR laser that was driven by an AC signal with amplitude voltage of 10 volts. The optical power of the laser was about 4.5$\mathrm{mW}$. It is noticed that this illumination system is capable of generating a large number of free carriers during the defect filling process, resulting in an efficient filling of defects, and a significantly large transient signal. Figure 18 shows the block diagram of the I-DLTS system.

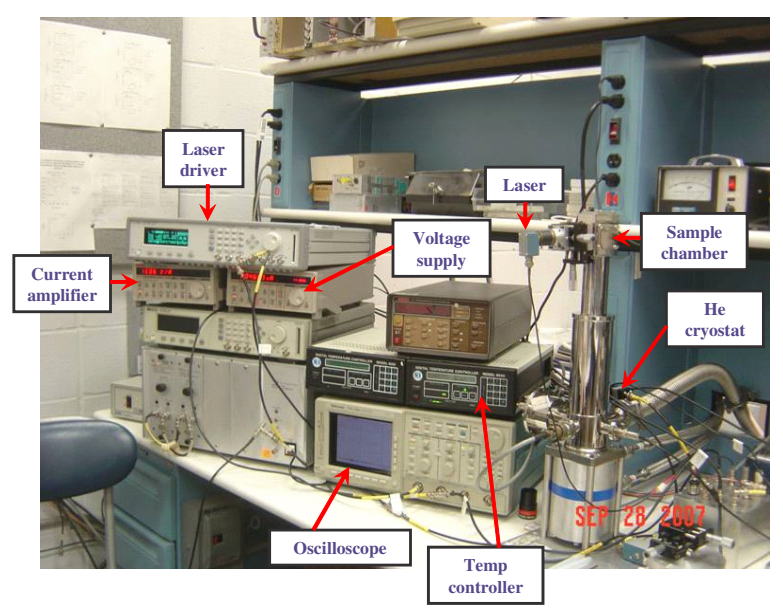

Figure 17. BNL's I-DLTS System

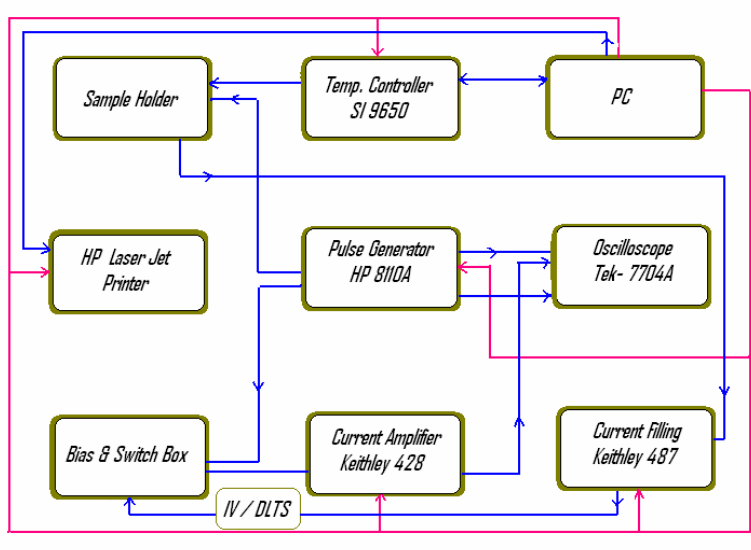

ILDTS Block Diagram

Figure 18. Block diagram of the I-DLTS System 
In our I-DLTS measurements, the detectors were cooled down to $10 \mathrm{~K}$, and then heated at a constant rate up to about $300 \mathrm{~K}$. The ramp-up temperature step was about $1 \mathrm{~K}$. At each temperature, the defect levels in a sample were first filled at 0 bias voltage by the $1030 \mathrm{~nm}$ laser driven by an AC pulse with a pulse width of $2 \mathrm{~ms}$ and a pulse period of $45 \mathrm{~ms}$. The defect filling time in a laser pulsing cycle is thus $2 \mathrm{~ms}$. Then, during the time when the laser was off in a laser pulsing cycle ( $45 \mathrm{~ms}-2 \mathrm{~ms}=43 \mathrm{~ms}$ ), a bias voltage was applied to the sample to efficiently detrap the charges from previously filled defect levels. The resulting transient current from this detrapping process was recorded by a fast oscilloscope and a PC during the laser-off period in the laser pulsing cycle. The I-DLTS signals were obtained by sampling the differences in the current transient at two sampling times: $t_{1}$ and $t_{2}$ at each temperature. The sampling time $t_{1}$ was increased in steps from $0.2 \mathrm{~ms}$ to $3 \mathrm{~ms}$, while the sampling time $\mathrm{t}_{2}$ was taken as $4 \mathrm{t}_{1}\left(\right.$ or $_{2}=\beta \mathrm{t}_{1}$, with $\left.\beta=4\right)$. Near a specific temperature, a certain defect level will emit the trapped charge, resulting in a peak, $\delta I_{D L T S}^{m}$, in the I-DTLS signal spectrum at this temperature, $T_{m}$, as shown in Figure 19.
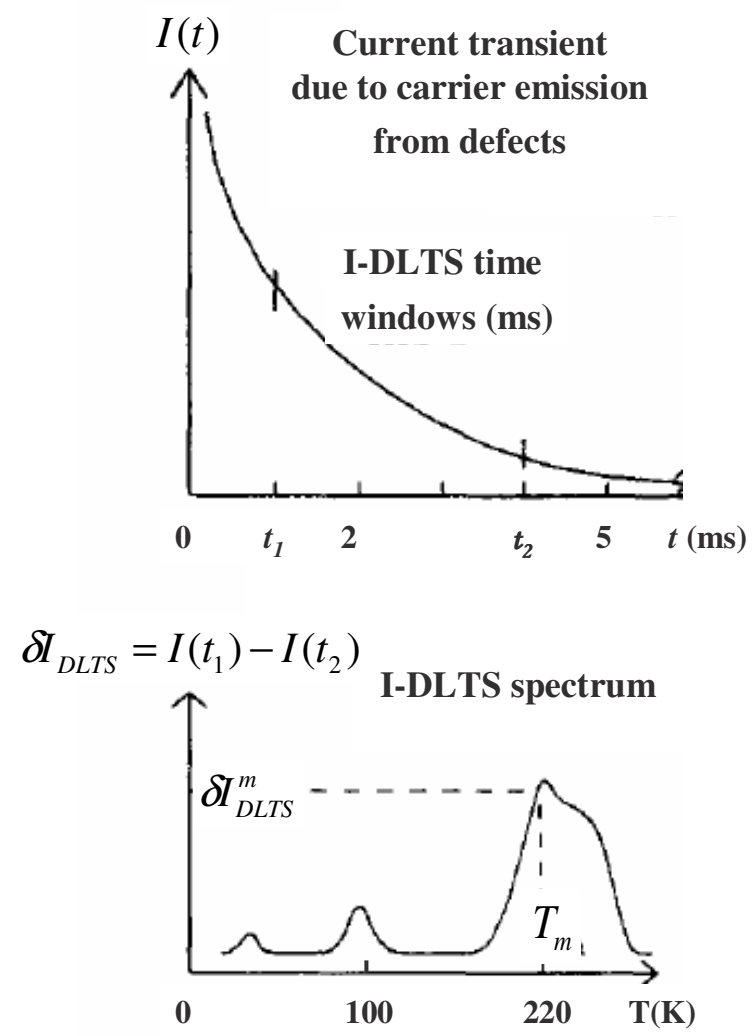

Fig. 3

Figure 19. Schematics of the DLTS principles.

The Arrhenius plot of $\log \left(T_{m}{ }^{2} t_{1}\right)$ vs. $1000 / T_{m}$ gives the information about the trap level:

$$
\left\{\begin{array}{l}
\Delta E=\frac{\text { slope }}{5.03} \\
\sigma=6.10 \times 10^{-22} \times 10^{-I}
\end{array}\right.
$$


where $\Delta E=E_{C}-E_{t}$ or $E_{t}-E_{V}$ is the energy level of the defect in the band gap, slope and the intersection $I$ are obtained from the Arrhenius plot, and $\sigma$ is the carrier trapping cross section.

The trap concentration $N_{t}$ can be obtained from the DLTS peak value $\delta I_{D L T S}^{m}$ :

$N_{t}=\frac{5.47 \delta I_{D L T S}^{m}}{q A W} t_{1}$

where $q$ is the electronic charge, $A$ the detector area, and $W$ the detector depletion depth.

More details of I-DLTS principles and modeling can be found in reference ${ }^{3}$. The energy levels, calculated from the I-DLTS signal, are measured from the edge of the Si conduction band or valence band.

To have systematic study the defects generated by $1 \mathrm{MeV}$ neutron irradiation in Geiger photodiodes for APeak Inc., and we placed some low resistivity (16-120 $\Omega-\mathrm{cm}$ ) BNL Si diodes in standard I-DLTS size $\left(0.1\right.$ to $0.25 \mathrm{~cm}^{2}, 300 \mu \mathrm{m}$ thick) together with APeak's Geiger photodiodes. Each BNL diode receives the same neutron fluence as one corresponding APeak's Geiger photodiodes. Both irradiated APeak's Geiger photodiodes and BNL diodes were measured by IDLTS from 13-300 K at BNL. However, due to the extremely low resistivity of the APeak's Geiger photodiodes, and non-standard sizes and configuration that are not optimal for I-DLTS measurements, we could only observer the peaks at very low temperatures $(<50 \mathrm{~K})$ in I-DLTS spectrum, which are due to shallow doping levels of phosphorous and boron. Fortunately, neutron radiation induced defect levels can be observed and measured in the shadowing low resistivity $\mathrm{Si}$ diodes. Since the type and concentration of the main neutron radiation induced defect levels in $\mathrm{Si}$ are not sensitive to the initial doping density, we can use the use the defect analysis on the low resistivity Si diodes to map the defects created in the APeak's Geiger photodiodes. Only the P-V (phosphorous-vacancy) center may have some dependence on the initial doping. But with the usage of a low resistivity Si diode, the this effect can be also minimized since the $[\mathrm{P}-\mathrm{V}]<<[\mathrm{P}]$ in our samples at the radiation fluence level used in this study.

Figure 21 shows the I-DLTS spectra of a irradiated low resistivity Si diode measured with various t1's. The Arrhenius plot of $\log \left(T_{m}{ }^{2} t_{l}\right)$ vs. $1000 / T_{m}$ can give the defect energy level in Si, and Eq. (2) can give the defect concentration. We note here that some I-DLTS peaks are complex of many defect levels (e.g. peak between 150-200 K, and between 220-300 K), the energy levels obtained are those of the dominated ones with influences of nearby levels. Some other peaks are too small for good analysis (e.g. peaks between 30-100 K). Higher radiation fluences may be needed to resolve these levels.

Table 20 shows the summary of the results of the defect analysis of the I-DLTS data. There are five peaks clearly observable in the I-DLTS spectra. The concentration of P impurity doping is $4.04 \times 10^{13} \mathrm{~cm}^{-3}$, corresponding to a resistivity of $100 \Omega-\mathrm{cm}$, which is within the initial specification of 16-120 $\Omega-\mathrm{cm}$. The concentrations of the A-center (oxygen-vacancy, or O-V), doubly-charged double vacancy center ( $\mathrm{V}-\mathrm{V}(--/-))$, and the E-center $(\mathrm{P}-\mathrm{V})$ are very similar, in the order of about $1 \times 10^{12} \mathrm{~cm}^{-3}$. The highest concentration is that of the singly-charged double-vacancy $(\mathrm{V}-\mathrm{V}(-/ 0))$, 
at about $2.1 \times 10^{12} \mathrm{~cm}^{-3}$. Within the complex peak between $220-300 \mathrm{~K}$, there may be some contributions by the extended defects, or defect clusters. ${ }^{4}$

Table 20. Defect levels in neutron-irradiated low resistivity Si diodes

\begin{tabular}{|l|l|l|l|l|l|}
\hline Defect level & $\mathrm{P}$ & $\begin{array}{l}\text { A-center } \\
(\mathrm{O}-\mathrm{V})\end{array}$ & $\mathrm{V}-\mathrm{V}(--/-)$ & $\mathrm{V}-\mathrm{V}(-/ 0)$ & $\begin{array}{l}\text { E-center }(\mathrm{P}-\mathrm{V}) \\
\text { and / or clusters }\end{array}$ \\
\hline $\mathrm{E}_{\mathrm{t}}(\mathrm{eV})$ & 0.05 & 0.16 & 0.23 & 0.38 & 0.45 \\
\hline $\mathrm{N}_{\mathrm{t}}\left(\mathrm{cm}^{-3}\right)$ & $4.04 \times 10^{13}$ & $1.02 \times 10^{12}$ & $1.17 \times 10^{12}$ & $2.11 \times 10^{12}$ & $1.05 \times 10^{12}$ \\
\hline
\end{tabular}

$\mathrm{T} 1(\mathrm{~s})$

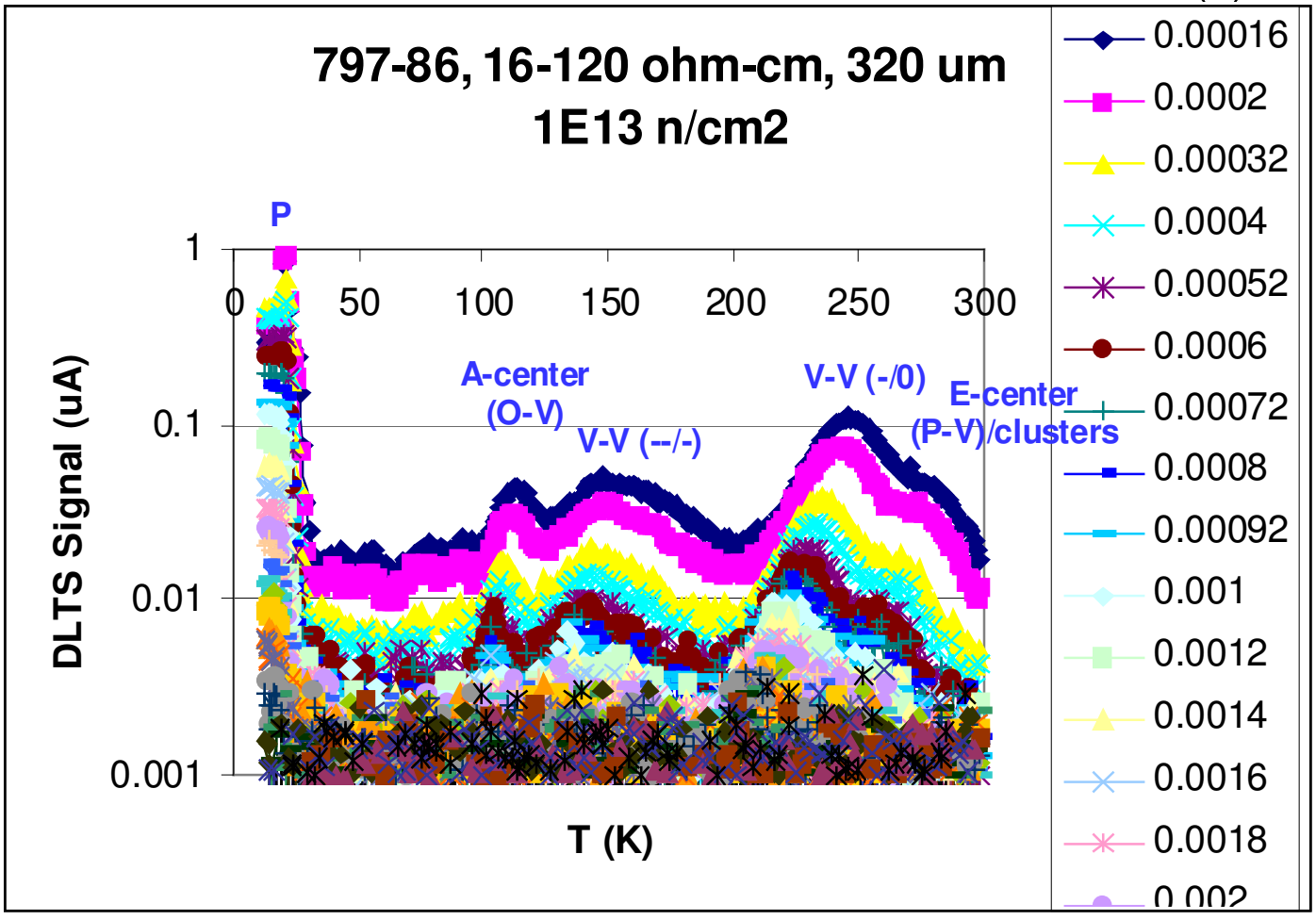

Figure 21. DLTS spectra.

\section{B.4 Main conclusions of the Phase I}

1. A new readout specific for microvertex applications has been developed;

2. Signal differentiation will require implementing memory/ capacitive storage cells at pixel level that may increase the pixel size. This more complex architecture will require an increased pixel size. In order to target competitive pixel size (10um) a new scaled down technology should be used. At the time of the report we have not identified such technology;

3. Irradiation with neutrons shows minor changes of the breakdown voltage;

4. Thermal treatment at $150 \mathrm{C}$ for 24 hours is capable of annealing most of the damage induced by neutrons;

5. In APGT pixels, DE is high even after 20-30\% residual damage on single junctions. However, we should expect a degradation of the positions resolution is DE for each junction is too low. More work has to be performed to better understand how DE of individual Geiger junctions will affect the position resolution; 
6. Even if we have not identified an off-the-shelf technology offered in multi-project wafer (MPW) suitable for APGT smaller pixel size fabrication and matching Phase I /Phase II budgets, the advantages of the high gain Geiger junctions for high resolution tracking should be further explored. Of course, custom technology development is an alternative solution but it does not fit the current budget for Phase I/Phase II SBIR. If Phase I/PhaseII SBIR contract budgets will be suitably updated to reflect current cost of performing R\&D in US, the development of a custom process should be worth re-visiting.

\section{DEVELOPED PRODUCTS}

\section{C.1 Publications}

None

\section{C.2 Procedures and protocols}

Readout circuit for Geiger pixels

C.3 Conferences/Proceedings

None

\footnotetext{
${ }^{1}$ S. Kleinfelder, S. Lim, X. Liu, and A. El Gamal, “A 10000 Frames/s CMOS Digital Pixel Sensor”, IEEE Journal of Solid-State Circuits, 36, p. 2049-2058, 2001.

${ }^{2}$ Y. Tomita, et al., Nucl. Instr. and Meth. A 270 (1988) 403.

3 Z. Li, "Systematic modeling and comparisons of capacitance and current-based microscopic defect analysis techniques for measurements of high-resistivity silicon detectors after irradiation" Nuclear Instruments and Methods in Physics Research A 403 (1998) 399-416

${ }^{4}$ Z. Li, H. W. Kraner, C. J. Li, B. Nielson, H. Feick, and G. Lindstroem; "Microscopic Analysis of Defects in a High Resistivity Silicon Detector Irradiated to 1.7 x $10^{15} \mathrm{~cm}^{2}$, IEEE Trans. Nucl. Sci. NS-43, 1590 (1996).
} 